\title{
Optic Nerve Metastasis from Breast Carcinoma after Treatment with Taxanes
}

\author{
Mónica Asencio-Duran'1, José-Luis Vallejo-Garcia1, Isabel Rodriguez-Rodriguez², \\ Margarita Sánchez-Orgaz ${ }^{1}$, Álvaro Arbizu-Duralde ${ }^{1}$ \\ ${ }^{1}$ Ophthalmology Department, Hospital La Paz, Madrid, Spain \\ ${ }^{2}$ Radiation Oncology Department, Hospital La Paz, Madrid, Spain \\ Email: masedur@hotmail.com
}

Received 12 April 2014; revised 9 May 2014; accepted 4 June 2014

Copyright (C) 2014 by authors and Scientific Research Publishing Inc.

This work is licensed under the Creative Commons Attribution International License (CC BY). http://creativecommons.org/licenses/by/4.0/

(c) (i) Open Access

\begin{abstract}
Introduction: The incidence of ocular metastasis from Breast Carcinoma is only between $5 \%-30 \%$, mainly located in choroid, but the location in optic nerve is even more infrequent. We present a rare case that combined both locations sequentially in the same eye. Methods: Case report. Results: A 58-year-old woman with advanced breast cancer was referred with a choroidal metastasis in her right eye which responded well to systemic Taxol and Avastin. Afterwards, she developed an optic nerve metastasis in the same eye when she was under the treatment, so it was changed to Docetaxel. Unfortunately the patient didn't respond to this treatment and died. Conclusions: Choroidal metastasis secondary to breast cancer generally responds well to radiotherapy. Even so, in cases that a great deterioration of visual acuity is expected after radiation, chemotherapy like taxanes is an alternative to preserve vision with complete tumor regression. Nevertheless, the presentation of a second metastasis in optic nerve can be indicative of inadequate treatment of metastatic disease or complication of the treatment, or both.
\end{abstract}

\section{Keywords}

Choroidal Metastasis, Optic Nerve Metastasis, Breast Cancer, Hormone Therapy, Taxanes

\section{Introduction}

Breast carcinoma is the most frequent tumor in women and the second reason of death in them. The incidence of ocular metastasis from breast cancer ranges between 5\% - 30\% [1] [2], with a 37\% of microscopic uveal affectation [2], whereas only $4.5 \%$ of all intraocular metastasis develop in the optic nerve, $43 \%$ of them coming from a primary breast neoplasia [3]. 
There are several treatment options for these metastasis, including observation, systemic treatment (cytotoxic, hormonal and inmunomodulatory agents), radiotherapy (external beam radiotherapy $=$ EBRT, or by means of plaques), local resection, photocoagulation, transpupillary termotherapy, cryotherapy or enucleation [1]. We present a rare case that combined both choroidal and optic nerve location sequentially in the same eye and that responded differently to treatment.

\section{Case Presentation}

A 58-year-old woman who was complaining of metamorphopsia was referred to our clinic. Her personal history was a left breast carcinoma treated 2 years before with radical mastectomy + limphadenectomy, chemotherapy and EBRT, with complete remission. At present she had multiple bone metastasis so that she was in the second cycle of systemic treatment with taxol and bevacizumab. The ophthalmic examination showed a visual acuity in right eye (RE) of 0.05 and 1 in left eye (LE), and the ophthalmoscopy revealed a yellowish posterior choroidal mass centered on the inferior temporal arcade that measured $11 \times 7.5$ millimeters in base. That mass was surrounded by exudative retinal detachment that was causing microfolds in the macula, and that was responsible of blurred vision (Figure 1). B-scan showed choroidal thickening measuring $11.7 \mathrm{~mm}$ in base and $2.5 \mathrm{~mm}$ in thickness with associated retinal detachment. A-scan showed moderate to high internal reflectivities with absence of calcium. The diagnosis of choroidal metastasis was made.

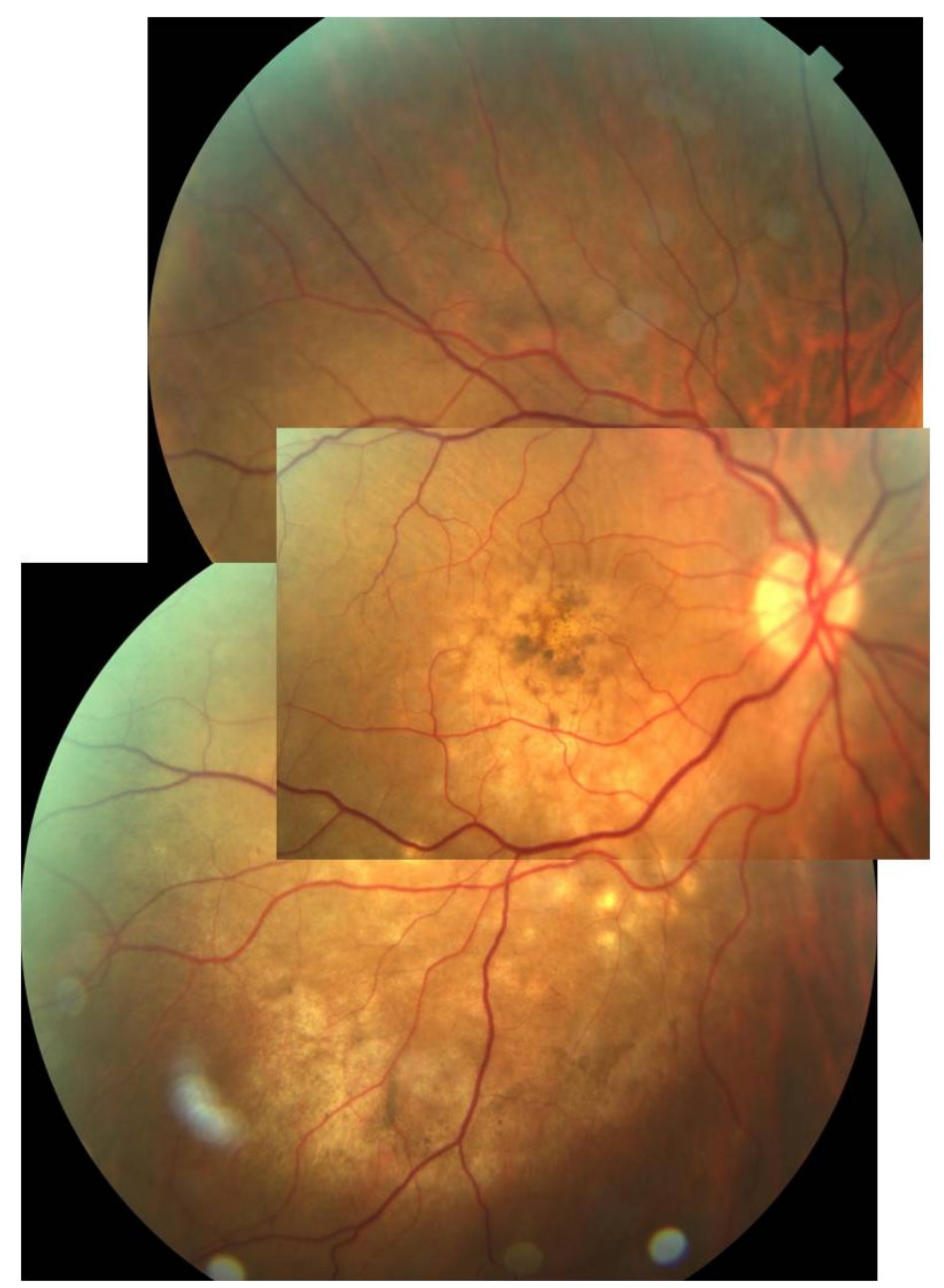

Figure 1. Retinography that shows the aspect of the right fundus of the patient at her arrival to the consultation: yellowish choroidal mass on the inferior temporal arcade that measured $11 \times 7.5$ millimeters in base, surrounded with exudative retinal detachment that was causing microfolds in the macula. 
Cycles of chemotherapy were continued until regression of the choroidal mass and complete visual recovery took place 13 months later. Nevertheless the patient continued the treatment due to other found metastasis. During a rest of the therapy due to bad general condition of the patient, a swelling of the optic disc was appreciated, and an MRI was performed. That revealed an increase in thickness in distal portion of the right optic nerve, compatible with metastasis. Treatment with Docetaxel was initiated, but three cycles after, the visual acuity in RE had lowered to no light perception, a complete afferent pupillary defect had developed and a mushromshaped elevation of the optic nerve head had appeared in the ophthalmoscopy, ultrasound and optic coherence tomography (OCT) (Figure 2). The patient was then sent for treatment with EBRT, but unfortunately, died 10 months later.

\section{Discussion}

The most supported treatment for ocular metastasis consists of treating the metastatic basal disease, specially if they are unresectables, the prognosis of life is limited and radiotherapy can threaten the vision as in case of posterior locations or the optic nerve. $25 \%$ - $40 \%$ of patients with breast cancer develop metastasis distantly in spite of treatment, which is considered to be incurable, but the palliative existing therapies obtain free-disease intervals that are in occasions very long. Today hormonal therapy is still preferred for women with positive estrogens-receptor [1], whereas the chemotherapeutic agents like taxanes (taxol and docetaxel), are preferred for tumors with negative receptor and with good results, specially in case of choroidal metastasis [4]. It is known that breast cancer has great capacity of dissemination, and that the incidence of taxanes-resistance in metastatic breast cancer is increasing [5]. Nevertheless it is possible that, as it is shown in our case, the taxanes could not protect from the long-term appearance of CNS (central nervous system) metastasis due to a minor penetration across the hematoencephalic barrier than other tissues. In addition, one could speculate on the possibility that these agents could have an inducting paper of metastasis to the brain and leptomeninges, as suggested by other authors, in whose case survival reduces dramatically from 3 to 13 months [6].

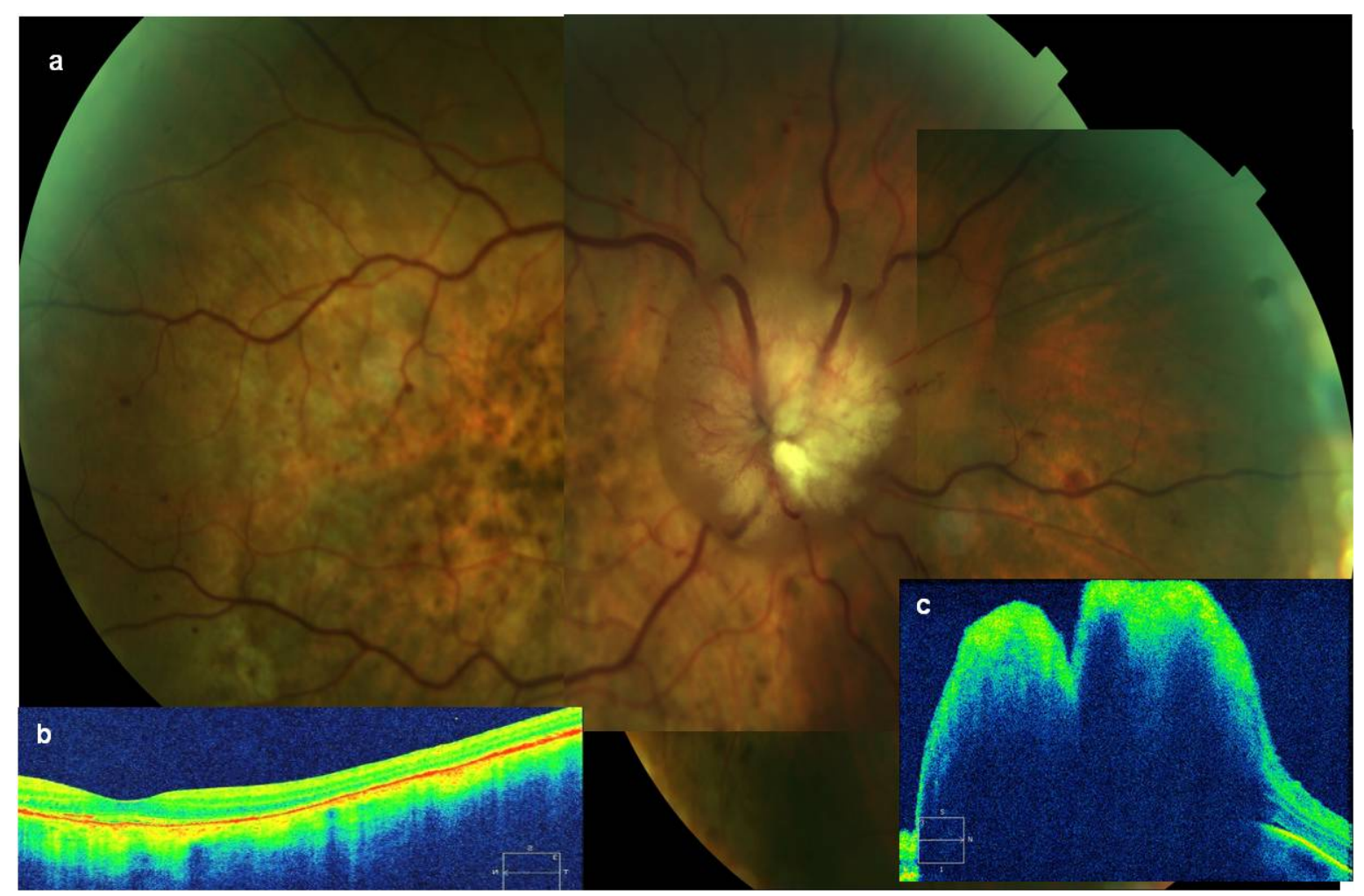

Figure 2. (a) Retinography that shows great prominence of the optic nerve head with edema, vascular erasing and retinal hemorrhages around, with complete regression of choroidal metastasis; (b) Optic coherence tomography (OCT) that shows foveal profile recovered and (c) tumour elevation on the optic nerve head. 


\section{Conclusion}

Up to now, there is no documented case of optic nerve metastasis after treatment with taxanes, that in addition (as it happened with our patient), proved to be successful for her choroidal metastasis. CNS metastasis has very important implications for these kinds of patients, since they are associated to a dramatic decrease in survival. It is necessary to continue looking for effective therapies that could protect from this complication to provide the best possible condition for these patients. We also would advise to carry out periodic ophthalmic controls in prolonged treatments with these agents, still in absence of documented ocular metastasis.

\section{References}

[1] Manquez, M.E., Brown, M.E., Shields, C.L. and Shields, J.A. (2006) Management of Choroidal Metastases from Breast Carcinomas Using Aromatase Inhibitors. Current Opinion in Ophthalmology, 17, 251-256. http://dx.doi.org/10.1097/01.icu.0000193105.22960.f6

[2] Biswas, J., Ho, T.C. and Bhavsar, K. (2007) Bilateral Metastasis to the Retina, Choroids and Optic Nerve from Breast Cancer: A Clinicopathological Case. Indian Journal of Ophthalmology, 55, 71-72. http://dx.doi.org/10.4103/0301-4738.29503

[3] Shields, J.A., Shields, C.L. and Singh, A.D. (2000) Metastatic Neoplasms in the Optic Disc: The 1999 Bjerrum Lecture: Part 2. Archives of Ophthalmology, 118, 217-224. http://dx.doi.org/10.1001/archopht.118.2.217

[4] Kosmas, C., Malamos, N.A. and Antonopoulos, M. (2000) Complete Regression of Choroidal Metastases from Breast Cancer after Docetaxel-Based Systemic Chemotherapy. Medical and Pediatric Oncology, 34, 229-230. http://dx.doi.org/10.1002/(SICI)1096-911X(200003)34:3<229::AID-MPO16>3.0.CO;2-U

[5] Zelek, L., Barthier, S., Riofrio, M., Fizazi, K., Rixe, O., Delord, J.P., Le Cesne, A. and Spielmann, M. (2001) Weekly Vinorelbine Is an Effective Palliative Regimen after Failure with Anthracyclines and Taxanes in Metastatic Breast Carcinoma. Cancer, 92, 2267-2272. http://dx.doi.org/10.1002/1097-0142(20011101)92:9<2267::AID-CNCR1572>3.0.CO;2-Q

[6] Crivellari, D., Pagani, O., Veronesi, A., Lombardi, D., Nolè, F., Thürlimann, B., et al. (2001) International Breast Cancer Study Group. High Incidence of Central Nervous System Involvement in Patients with Metastatic or Locally Advanced Breast Cancer Treated with Epirubicin and Docetaxel. Annals of Oncology, 12, 353-356. http://dx.doi.org/10.1023/A:1011132609055 